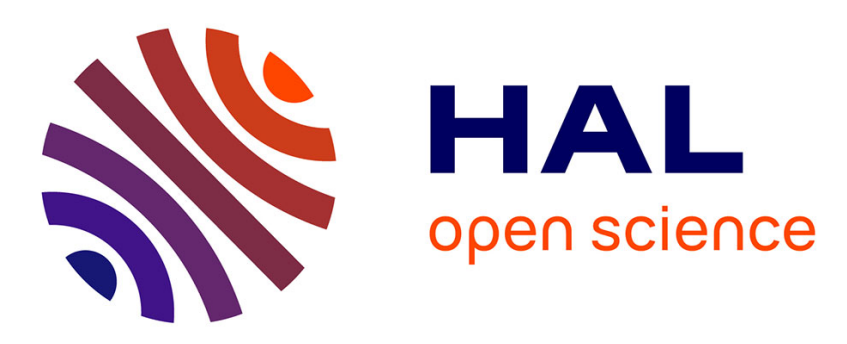

\title{
Interaction homme-machine et personnalisation des visites : enjeux et perspectives critiques
}

Stéphanie Rey, Christophe Bortolaso, Anke Brock, Célia Picard, Mustapha Derras, Nadine Couture

\section{- To cite this version:}

Stéphanie Rey, Christophe Bortolaso, Anke Brock, Célia Picard, Mustapha Derras, et al.. Interaction homme-machine et personnalisation des visites : enjeux et perspectives critiques. Culture et Musées, 2020, 35, pp.77-106. 10.4000/culturemusees.4527 . hal-02883368

\section{HAL Id: hal-02883368 \\ https://hal-enac.archives-ouvertes.fr/hal-02883368}

Submitted on 6 Jul 2020

HAL is a multi-disciplinary open access archive for the deposit and dissemination of scientific research documents, whether they are published or not. The documents may come from teaching and research institutions in France or abroad, or from public or private research centers.
L'archive ouverte pluridisciplinaire HAL, est destinée au dépôt et à la diffusion de documents scientifiques de niveau recherche, publiés ou non, émanant des établissements d'enseignement et de recherche français ou étrangers, des laboratoires publics ou privés. 


\title{
Interaction hommes-machine et personnalisation des visites : Enjeux et perspectives critiques
}

\author{
Stéphanie Rey, Christophe Bortolaso, Anke M. Brock, Célia Picard, \\ Mustapha Derras \& Nadine Couture
}

Stéphanie Rey, Berger-Levrault, Toulouse ; Université de Bordeaux LaBRI et ESTIA Institute of Technology, Bidart

Christophe Bortolaso, Berger-Levrault, Toulouse

Anke M. Brock, Université de Toulouse, ENAC

Célia Picard, Université de Toulouse, ENAC

Mustapha Derras, Berger-Levrault, Toulouse

Nadine Couture, Université de Bordeaux LaBRI et ESTIA Institute of Technology, Bidart

\section{Introduction}

La visite d'un musée est influencée par de nombreux critères liés aux identités multiples des visiteurs et aux contextes de leur visite (Eidelman, 2013 : 73-113). Le temps dont le visiteur dispose, ses connaissances préalables, les personnes avec qui il effectue sa visite, ses humeurs et envies du jour sont autant de facteurs qui impactent ses attentes et ses pratiques. Face à la «pluralité des figures de visiteurs » (ibid.) et de leurs pratiques de visite, les musées proposent différents types de médiation du contenu culturel. Les visites guidées permettent ainsi de proposer des parcours thématisés ou profilés selon de grandes catégories de visiteurs (familles, groupes, personnes en situation de handicap, tranches d'âge des enfants). Au cours de ces visites, les médiateurs culturels adaptent en temps réel le discours à leur auditoire et leurs réactions, dans un «travail de dialogue » qui construit un récit partagé d'interprétation (Gellereau, 2015 : 189). Cependant, ces visites ne sont disponibles qu'à des créneaux horaires précis et en quantité limitée. Certains guides de visite multimédias ou applications mobiles disponibles en permanence - permettent de fournir aux visiteurs des parcours de visite selon certaines thématiques et d'adapter le discours selon les âges des visiteurs. Les tablettes tactiles sont également utilisées, par exemple, pour fournir des contenus de visite adaptés aux enfants, qu'ils soient ou non en situation de handicap (Bougenies et al., 2015 : 115-139). Ces types de dispositifs renouvellent l'expérience de visite guidée thématisée ou profilée, mais ne permettent pas de proposer des parcours pleinement personnalisés, selon des combinaisons de caractéristiques propres aux visiteurs (au-delà de profils génériques ou de la seule thématique), ni de s'adapter en temps réel à leurs souhaits. Dès lors se pose la question suivante: les dispositifs numériques pourraient-il permettre d'aller plus loin dans la personnalisation, au-delà des visites profilées ou thématisées ? Et si oui, quels seraient les enjeux et les problématiques d'une telle personnalisation pour les professionnels des musées ?

Pour répondre à ces questions, nous nous sommes intéressés au processus de scénarisation de ces visites par les professionnels des musées, et plus particulièrement les médiateurs culturels. Nous mettons ainsi en place une démarche de conception centrée utilisateur $(\mathrm{CCU})^{1}$ où l'utilisateur est le médiateur dans sa tâche de création de parcours de visites personnalisées.

\footnotetext{
${ }^{1}$ Une méthode utilisée couramment en Interaction homme-machine, discipline à la croisée de l'informatique et des sciences humaines et sociales.
} 
Nous présentons dans une première partie un état de l'art et de l'existant de la personnalisation dans les musées. La deuxième partie présente l'approche centrée sur l'utilisateur d'un outil numérique et la méthode utilisée. Les résultats de l'étude menée dans les musées partenaires seront ensuite décrits. La troisième partie détaille ainsi les opportunités, enjeux et problématiques de la personnalisation numérique des parcours de visite extraits des entretiens. La quatrième partie définit les exigences pour la conception d'un outil d'aide à la scénarisation des parcours de visite, issues de l'observation prospective des stratégies de conception des médiateurs. En conclusion, nous reviendrons sur les transformations potentielles de la notion de personnalisation dans la sphère muséale.

\section{De la personnalisation des visites}

Les termes de customisation, personnalisation et individualisation sont souvent utilisés indifféremment pour se référer à la prise en compte des spécificités de l'utilisateur dans la mise en œuvre d'un produit numérique. Pourtant, ils renvoient à des concepts différents. Plusieurs définitions existent selon les auteurs, nous nous basons ici sur celles proposées par Bowen et Filippini-Fantoni (2004 : s.p.). La customisation correspond au choix explicite par l'utilisateur de paramètres lui permettant de configurer de manière active la présentation du contenu (par exemple, le choix de la langue, le type de contenu et son organisation). Par exemple, le Art Institute de Chicago propose Journey Maker, une application sur table tactile à l'entrée du musée (McHugh, 2017 : s.p.). Elle permet aux familles de construire un parcours sur mesure en choisissant une thématique parmi huit possibles (super héros, monstres, voyage dans le temps, etc.) puis une œuvre pour chacun des cinq chapitres de l'histoire correspondante. Ils déclarent ainsi proposer 262000 combinaisons différentes. Le parcours correspondant peut ensuite être imprimé sous forme de livret contenant des jeux sur les œuvres choisies, un plan du musée et des indications de guidage. Le visiteur construit entièrement son parcours en choisissant les œuvres, le système agit donc ici plutôt comme un cadre d'aide au choix dans un processus de customisation. La personnalisation, quant à elle, permet au système d'adapter automatiquement le contenu en fonction d'un profil utilisateur. Celui-ci est renseigné de manière explicite, par exemple par le remplissage de questionnaires (Roussou et al., 2013 : 547-552), ou implicite, en fonction de la navigation et des actions du visiteur in situ (Wecker, 2016: 978-983), sur des applications numériques (Antoniou \& Lepouras, 2010: $7: 2-7: 19$ ) ou sur les réseaux sociaux (Naudet et al., 2013: 67-72). L'individualisation, enfin, est un terme chapeau permettant de recouvrir ces différents concepts. Nous nous concentrons dans ce travail sur l'adaptation du contenu de la visite en fonction du profil d'un visiteur ou d'un groupe de visiteurs, et choisissons donc d'utiliser le terme de personnalisation de la visite.

Cette personnalisation peut s'initier en dehors du cadre physique du musée lors de la préparation de la visite. Le Musée du Louvre à Paris propose ainsi sur son site web de sélectionner quatre critères (thème, durée, jour, public) et d'imprimer la visite correspondante parmi les 32 visites proposées ${ }^{2}$. Cependant, toutes les combinaisons ne sont pas traitées et le système ne permet de choisir qu'une seule valeur pour le type de public parmi plusieurs tranches d'âges, compositions de groupe et situations de handicap. Il n'est donc pas possible, par exemple, de préparer une visite pour une famille avec des enfants d'âges différents et une personne en situation de handicap. Certains musées ont également tenté de proposer des expériences de visites personnalisées lors de la visite physique en elle-même. Ainsi, au Palais des Beaux-Arts de Lille, trois options sont proposées sur une table tactile à l'entrée du musée : 1) constituer son propre parcours, 2) choisir parmi une liste de visites thématiques préétablies

${ }^{2}$ Site web du musée du Louvre présentant les parcours de visite et les critères de choix https://www.louvre.fr/parcours consulté le 21/01/2020. 
(également visibles sur leur site web) ou 3) visualiser l'ensemble des œuvres du musée. Une fois le parcours créé ou choisi, les visiteurs peuvent demander à imprimer le livret de visite correspondant ou l'envoyer sur leur mobile pour suivre le parcours à l'aide de l'application du musée ${ }^{3}$. La création de parcours selon la première fonctionnalité se révèle complexe pour les visiteurs seuls qui ne savent pas par où commencer. Les parcours préétablis, quant à eux, ne prennent pas en compte l'évolution dynamique des collections du musée (prêts d'œuvres, travaux) et peuvent se révéler rapidement obsolètes. En pratique, une médiatrice culturelle présente à côté de la table tactile utilise la troisième fonctionnalité de visualisation des œuvres pour coconstruire le parcours avec les visiteurs ${ }^{4}$. Celle-ci peut ainsi les conseiller dans la constitution d'un parcours en les interrogeant sur leurs centres d'intérêt, la composition de leur groupe, le temps dont ils disposent, etc. Le numérique sert ici de support pour la médiatrice dans la constitution du parcours personnalisé, mais la tâche doit être répétée pour chaque nouveau groupe de visiteurs, sans pouvoir réutiliser ce qui a été créé précédemment.

La personnalisation est souvent traitée en recherche en informatique par la modélisation des utilisateurs et la génération automatique de contenu, par exemple dans les projets CHIP (Wang et al., 2008 : 283-290) et PEACH (Stock et al., 2007 : 257-304). Le projet Cultural Heritage Information Personalization (CHIP) propose ainsi une suite d'outils utilisant le web sémantique pour recommander des œuvres d'art et des visites personnalisées en fonction du profil des visiteurs. Le visiteur est tout d'abord invité à noter des œuvres de la collection numérique du Rijksmuseum (Amsterdam, Pays-Bas) sur l'outil web «Art Recommander». Chaque œuvre possède des métadonnées enrichies sémantiquement afin de les relier à des thèmes et à d'autres œuvres de la collection. Le profil du visiteur est ainsi constitué en inférant ses centres d'intérêt à partir des notes données aux œuvres. Un deuxième outil web, «Tour Wizard», propose alors au visiteur des visites personnalisées en fonction de son profil et de la longueur souhaitée pour le parcours (durée ou nombre d'œuvres maximum). Il peut suivre ce parcours dans le musée grâce au «Mobile Guide », une application sur PDA ${ }^{5}$, et l'adapter dynamiquement en modifiant la durée ou en notant de nouvelles œuvres. Personal Experience with Active cultural Heritage (PEACH) propose des contenus multimédias générés automatiquement en fonction des comportements des visiteurs. En arrivant au Buonconsiglio Castello (Trento, Italie), le visiteur choisit parmi plusieurs personnages animés sur un écran celui qui le guidera dans sa visite. Durant la visite, le système mobile affiche des contenus audios et vidéos générés automatiquement pour s'adapter en fonction de l'œuvre devant laquelle le visiteur se trouve (géolocalisation), du personnage choisi (perspective de la présentation), des œuvres déjà vues, du temps passé devant chacune, des notes qui leur ont été données pendant la visite et de la langue choisie. À la fin de la visite, un résumé personnalisé est généré automatiquement et imprimé. Il contient des informations sur les détails observés et des liens pour approfondir le sujet lors de la post-visite, sur internet ou dans des musées des alentours. Cependant ces solutions posent plusieurs problèmes. Les groupes de visiteurs ne sont ainsi pas pris en compte dans le profilage et ces outils manquent d'expérimentation avec des scénarii d'utilisation réels, la plupart des modèles étant testés sur des jeux de données simulés (Ardissono et al., 2012 : 73-99). Filippini-Fantoni (2009) souligne également des problèmes de doublons et d'incohérences lors de la génération automatique de contenus et de non-perception de la personnalisation par les visiteurs, leurs envies étant difficilement

\footnotetext{
${ }^{3}$ Site de l'application mobile du Palais des Beaux-Arts de Lille https://pba.lille.fr/Le-Palaisdes-Beaux-Arts-numerique consulté le 16/01/2020

${ }^{4}$ Communication orale au Palais des Beaux-Arts de Lille le 10/11/2018

${ }^{5}$ Un Personal Digital Assistant est un appareil numérique portable assurant les fonctions d'assistant personnel : gestion des contacts, de l'agenda, des courriers, etc.
} 
interprétables par les algorithmes (Andreacola, 2014 : sp). Ces solutions s'abstraient en outre des compétences des personnels des musées pour la création de contenus culturels adaptés à leurs visiteurs.

La création de parcours de visites personnalisées par les professionnels des musées est également traitée en recherche en interaction homme-machine. Ce domaine étudie la relation entre utilisateur et système, et cherche à accompagner numériquement les compétences spécifiques des utilisateurs dans la réalisation d'une tâche. Le projet européen Cultural Heritage Experiences through Socio-personal interactions and Storytelling (CHESS) ${ }^{6}$ développe ainsi une recherche à la fois sur l'expérience de visite narrative personnalisée pour les visiteurs et sur la création de ces visites par les experts du musée (Vayanou et al., 2014 : 37-48). Du côté des visiteurs, un court questionnaire portant sur les préférences culturelles (type de film et lecture pour les adultes, activité et type de personnage pour les enfants) permet d'attribuer au visiteur un «persona $»^{7}$ (Roussou et al., 2013 : 547-552) parmi cinq identifiés pour le Musée de l'Acropole (Athènes, Grèce) ou deux pour la Cité de l'Espace (Toulouse, France) (Figure 1). L'application sur tablette propose alors au visiteur de suivre une histoire en fonction du «persona » lui correspondant le plus. Celle-ci est composée de plusieurs étapes face à différents expôts et peut être enrichie de vidéos, d'images et de jeux en réalité augmentée ${ }^{8}$. Le visiteur est guidé à l'aide d'indications visuelles au Musée de l'Acropole et sur le plan du musée grâce à la géolocalisation à la Cité de l'espace. Du côté des spécialistes du contenu, un outil d'édition leur permet de créer chaque portion d'histoire, de les associer à des points d'intérêts dans le musée et à des contenus multimédias, et de les organiser dans un graphe permettant de gérer les choix des visiteurs au cours de la visite (Vayanou et al., 2014 : 37-48). Cependant l'utilisation de personas ne permet pas de prendre en compte les groupes de visiteurs, alors que la visite de musée est souvent une activité sociale (Hood, 1983 : 50-57).

Figure 1: le projet CHESS au Musée de l'Acropole (Athènes, Grèce). À gauche le questionnaire pour les enfants (extrait de Roussou \& Katifori, 2018. (C) CC BY 4.0, Roussou et Katifori, https://www.mdpi.com/2414-4088/2/2/32). À droite un exemple de persona (extrait de Roussou et al., 2013. (C) Roussou, Katifori, Pujol, Vayanou, Rennick-Egglestone).

\footnotetext{
${ }_{6}^{6}$ Vidéo de présentation du projet CHESS https://www.youtube.com/watch?v=fZRiE7VR$\underline{x w \& f e a t u r e=y o u t u . b e}$

7 «Les personas sont des représentations fictives, spécifiques et concrètes des utilisateurs cibles » (Adlin \& Pruitt, $2010:$ 1, traduction des auteurs).

8 La réalité augmentée «a pour but d'enrichir la perception et la connaissance d'un environnement réel par l'ajout d'informations numériques le concernant. Ces informations sont le plus souvent visuelles, parfois sonores, rarement haptiques. ». (Arnaldi et al., 2018 :
} 

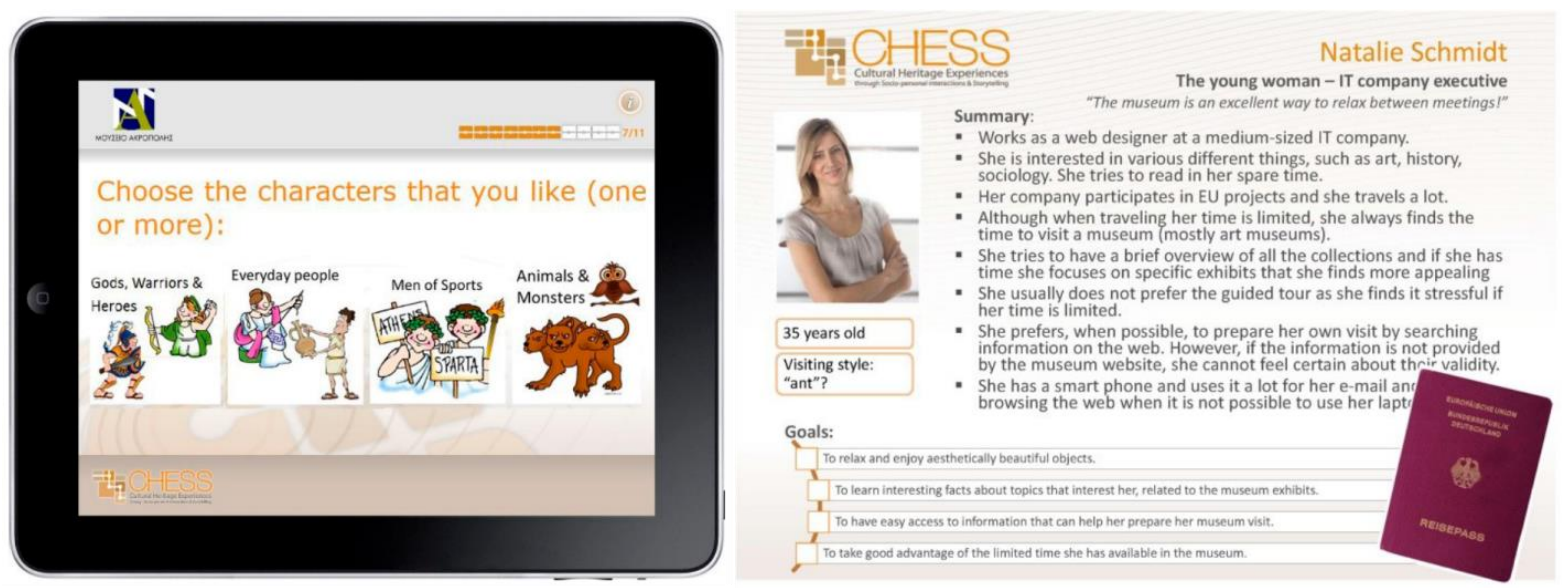

Le projet «meSch» (Petrelli et al., 2013 : 58-63) s'est appliqué quant à lui à fournir une expérience augmentée aux visiteurs à travers l'utilisation d'artefacts tangibles interactifs ${ }^{9}$. Lors d'une des expérimentations au Museon (La Hague, Pays-Bas), les visiteurs peuvent choisir parmi six fac-similés celui qui les accompagnera pendant leur visite (Figure 2). L'objet choisi détermine la langue (néerlandais ou anglais) et le point de vue de la narration (soldat, civil ou fonctionnaire). Différentes stations interactives disséminées dans l'exposition permettent de jouer un contenu personnalisé lorsque le visiteur place l'objet sur le socle prévu à cet effet ${ }^{10}$. La comparaison avec une interface de choix sur un téléphone portable a démontré la préférence des utilisateurs pour les interactions tangibles (Petrelli et O'Brien, 2018 : 1-12). Le consortium européen a également fourni des outils et des méthodes pour permettre aux conservateurs de construire les objets interactifs, les scénarios et les contenus associés (Not \& Petrelli, 2018 : 3-19). Cependant, la personnalisation est ici limitée à un petit nombre de caractéristiques : la langue et le point de vue de la narration.

Figure 2 : le projet meSch au Museon (La Hague, Pays-Bas), à gauche les artefacts tangibles et à droite une station interactive activée avec le «brassard du fonctionnaire». Images extraites de (Petrelli et O’Brien, 2018), (C Petrelli et O’Brien.
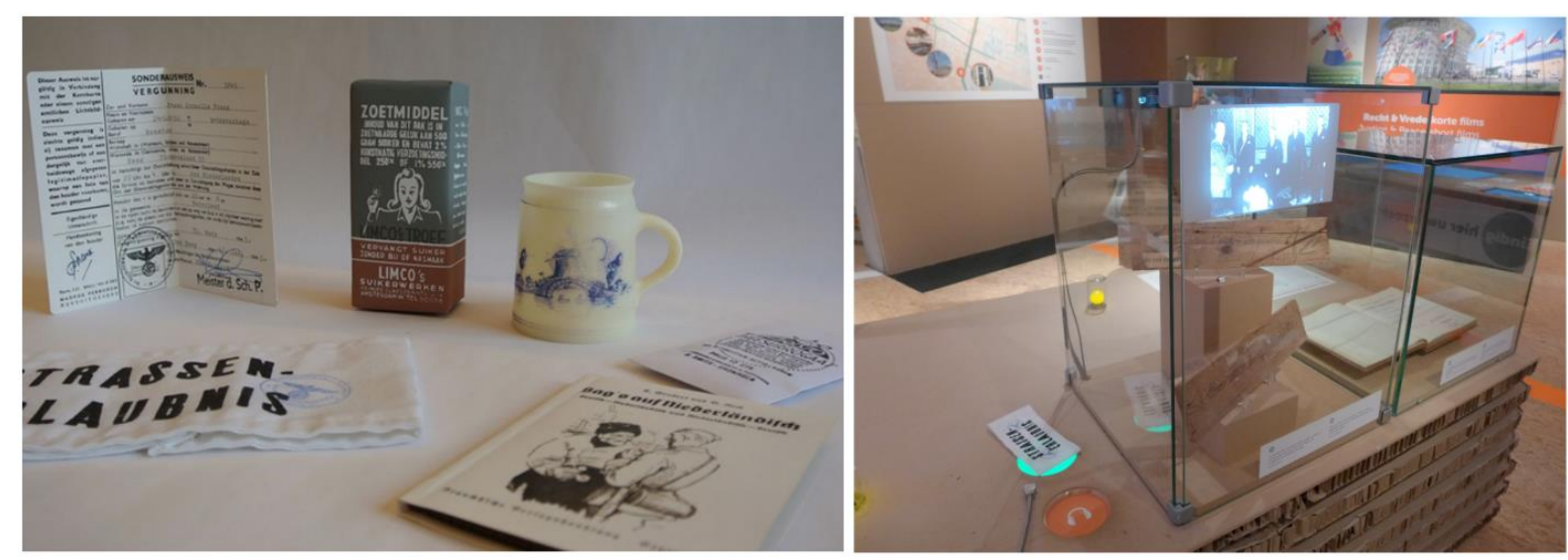

9 Les interfaces utilisateurs tangibles (TUI) sont définies comme «des représentations physiques d'informations numériques et des moyens de les contrôler, permettant littéralement aux utilisateurs d'attraper les données avec leurs mains ». (Shaer \& Hornecker, 2010:4, traduit par les auteurs)

10 Techniquement, des lecteurs RFID sont utilisés pour repérer les objets sur les socles. L'identification par radiofréquence (RFID) utilise des champs électromagnétiques pour identifier des étiquettes fixées sur des objets. 
De cet état de l'art, nous retenons que les solutions de personnalisation proposent des contenus essentiellement thématisés ou profilés, qui ne considèrent pas des niveaux plus fins comme la composition des groupes de visiteurs. Nous proposons dans le chapitre suivant une méthodologie d'enquête nous permettant d'étudier comment dépasser ces limites.

\section{L'interaction homme-machine et la conception centrée utilisateur}

\section{Le domaine de l'interaction homme-machine}

L'interaction homme-machine (IHM) est définie par l'Association for Computing Machinery $(\mathrm{ACM})$ comme « la discipline qui se consacre à la conception, l'évaluation et la mise en œuvre de systèmes informatiques interactifs pour l'usage humain ainsi qu'à l'étude des phénomènes majeurs qui les entourent» (Hewett et al., 1992: 5, traduit par les auteurs). L'IHM, par son sujet d'étude, est un domaine interdisciplinaire. " Parce que l'IHM étudie la communication entre un humain et une machine, elle s'appuie sur des connaissances complémentaires tant du côté de la machine que de l'humain. Du côté de la machine, les techniques d'infographie, les systèmes d'exploitation, les langages de programmation et les environnements de développement sont concernés. Sur le plan humain, la théorie de la communication, les disciplines du design graphique et industriel, la linguistique, les sciences sociales, la psychologie cognitive et l'étude de la performance humaine sont pertinentes. Et, il faut bien sûr également considérer les méthodes d'ingénierie et de conception » (ibid.). Cette discipline intègre donc des méthodes d'analyse et de conception inspirées de l'ethnographie et des sciences sociales afin d'étudier les comportements humains pour proposer les outils numériques et les interactions avec ceux-ci les plus adaptés.

La communauté de recherche en IHM s'est souvent intéressée au domaine culturel et plus particulièrement aux musées, en témoigne le récent livre de Hornecker et Ciolfi (2019). Il recense des projets en réalité virtuelle ou augmentée (ibid. : 42-54) pour l'apprentissage, souvent historique, la reconstruction virtuelle ou la superposition de contenu culturel aux expositions à travers des smartphones, tablettes ou des casques. Les interfaces utilisateurs tangibles (TUI) sont également très présentes dans la recherche en IHM appliquée à la culture. Les TUI consistent en l'utilisation d'objets physiques équipés numériquement et permettent de favoriser la collaboration et la conscience mutuelle dans les groupes de visiteurs (Hornecker \& Stifter, 2006: 135-142). Ainsi Hornecker et Stifter notent: «notre étude souligne le rôle des supports et des interfaces physiques pour attirer une diversité de visiteurs de tous âges et groupes d'intérêt. Elle met également en évidence les effets des différentes configurations physiques des installations sur les constellations émergentes de visiteurs autour d'elles et sur leurs modes d'interaction ». Cette étude est étayée par des travaux théoriques qui offrent notamment un cadre conceptuel pour penser les effets sociaux des interfaces physiques et matérielles à travers quatre thèmes: la qualité tactile de la "manipulation tangible », l'appropriation de l'espace au travers des « interactions spatiales », la «facilitation incarnée » qui impacte la collaboration au sein des groupes et «l'expressivité des représentations » tangibles ou incarnées (Hornecker \& Buur, 2006 : 437-446). La plupart des travaux en IHM concerne l'interaction du point de vue des visiteurs, et s'attache à évaluer entre autres leur compréhension, leur satisfaction ou leur apprentissage au travers de questionnaires semiouverts, d'analyses vidéo, de fichiers de traces informatiques, etc. (id. : 105 sqq.). Damala et. al ont ainsi proposé le modèle MUSETECH, un cadre complet pour l'évaluation de la technologie avant et après son introduction dans un musée, qui prend en compte le triple point de vue des professionnels du musée, des institutions culturelles et des visiteurs (2019: 1-22).

\section{La méthode de conception centrée utilisateur}


Une des méthodes fondamentales en IHM est la CCU. Cette méthode a été décrite pour la première fois dans un ouvrage édité par Norman et Draper en 1985. Elle est ainsi définie : « la conception centrée sur l'utilisateur place l'utilisateur au centre du processus de conception, depuis l'analyse initiale des besoins des utilisateurs jusqu'aux tests et à l'évaluation » (Beaudouin-Lafon \& Mackay, 2003 : 1009). En 1999, une norme internationale ISO $13407^{11}$ a été introduite pour formaliser le «processus de conception centrée sur l'opérateur humain pour les systèmes interactifs ». Cette norme a ensuite été révisée par la norme ISO 9241-210 ${ }^{12}$ en 2010. Elle précise les critères de mise en œuvre de la démarche CCU : la compréhension en amont des utilisateurs, de leurs tâches et de leur environnement ; l'implication active des utilisateurs tout au long du développement du produit ; l'itération entre les différentes phases du processus de conception ; la constitution d'une équipe de conception pluridisciplinaire ; la répartition appropriée des fonctions entre les utilisateurs (habitudes, personnalités, compétences) et la technologie (performance, fonctionnalités). Le cycle itératif de conception est composé de quatre grandes étapes: 1) la compréhension de l'activité, du contexte d'utilisation, 2) la compréhension des exigences et des tâches des utilisateurs, 3) la conception et le maquettage de solutions, 4) l'évaluation de ces solutions auprès des utilisateurs (Lallemand \& Gronier, $2018: 31$ ).

Nous avons utilisé une approche de CCU afin de mieux prendre en compte les besoins, les compétences et les comportements des professionnels des musées pour la création de parcours de visite personnalisés. Sur une période de neuf mois, nous avons mené une analyse des besoins auprès de quatre institutions culturelles (la Cité du Vin et Cap Sciences à Bordeaux, le Musée Saint-Raymond et le Musée des Augustins à Toulouse). Nous avons tout d'abord conduit des entretiens généraux auprès de professionnels de profils variés, que nous avons approfondies par des entretiens semi-directifs consacrés à la création de visites avec des spécialistes du service des publics. Nous avons ensuite complété cette étude par l'observation d'un exercice prospectif in situ de création de visites personnalisées dans deux musées.

\section{Opportunités et enjeux de la personnalisation numérique}

Les premiers entretiens ont impliqué dix personnes occupant une grande variété de postes : directeurs de musées, conservateurs, médiateurs culturels, professionnels de la communication et responsables informatique. La discussion était guidée par une grille de questions portant sur trois thèmes principaux : l'organisation du musée, les visiteurs et leurs comportements, la collection et les visites. L'objectif était de mieux comprendre le domaine muséal, d'identifier les défis et les besoins des musées et de cibler les acteurs avec lesquels poursuivre l'analyse. Nous avons ainsi constaté que, pour la plupart des institutions interrogées, donner à l'exposition permanente une perspective nouvelle et attractive est un enjeu fort, afin notamment d'inciter les visiteurs à revenir et de contrer l'effet «stadium » des expositions temporaires décrit par Bradburn (2001: 75-76). Nous avons également noté qu'une fois l'exposition permanente mise en place, le service de médiation est chargé d'organiser des activités et des visites pour tous les types de visiteurs (écoles, familles, groupes, etc.). Nous avons donc choisi de continuer notre enquête avec les médiateurs culturels qui ont l'habitude de créer des parcours adaptés aux visiteurs et au contexte de la visite. Cela rejoint les considérations de Davallon (2010:236) qui enjoint d'intégrer des

\footnotetext{
${ }^{11}$ International Organization for Standardization. (1999). ISO 13 407:1999, Human-centered design processes for interactive systems

${ }^{12}$ International Organization for Standardization. (2010). ISO 9241-210:2010, Ergonomics of human-system interaction - Part 210: Human-centered design for interactive systems.
} 
spécialistes prenant en compte les visiteurs dans la création du « schéma scénationnel » d'une exposition, i.e. l'anticipation de tous les différents parcours et expériences possibles des visiteurs.

Nous avons donc approfondi cette analyse globale par des entretiens semi-directifs (Lallemand \& Gronier, 2018: 113) avec deux médiateurs culturels (M1 et M2) et deux responsables du service des publics (R1 et R2) de trois des institutions précédentes. Le but de ces entretiens était de comprendre le processus actuel de création de visites guidées et les souhaits pour de futures visites idéales. Dans la première partie de l'entrevue, nous avons demandé aux spécialistes de décrire leur dernière expérience de création d'une visite guidée, ainsi que sa conduite effective dans le musée et l'adaptation dynamique de leur discours au public lors de celle-ci. Le but était de faire émerger les caractéristiques d'adaptation courantes. Dans la deuxième partie, les spécialistes ont été invités à imaginer la visite idéale pour leurs visiteurs futurs et à en détailler les caractéristiques.

Pour tous les médiateurs interrogés, la future visite idéale s'adapte aux désirs, besoins et envies des visiteurs. «A priori on peut dire que le parcours idéal, c'est celui que les gens se feraient, mais ils n'arrivent pas à le choisir parce qu'ils n'ont pas peut-être assez d'informations sur comment le choisir»(R1). Ainsi, pour cette responsable culturelle, il faudrait un système «qui leur donne les capacités de choisir, un peu à la carte, c'est-à-dire [...] j'arrive effectivement j'ai qu'une heure [...] et je m'intéresse absolument à ça. Je pense que le côté un peu à la carte ce serait la manière idéale parce que ça répondrait à tous les publics, et pour moi ce serait vraiment dans une optique de médiation. [...] Dans l'idéal un parcours, qu'il soit accompagné ou non humainement, il devrait pouvoir s'adapter». Cela implique la création de nombreux parcours de visite selon M2: «C'est à dire qu'il faut que ce soit un truc complètement subjectif par les personnes qui créent l'application ou le truc de visite, mais qui pourrait être démultiplié, c'est-à-dire que tu en aurais $10000 »$.

Ces parcours de visite doivent tout de même garder une certaine cohérence : selon R1 «le risque c'est $[\ldots]$ peut-être qu'à la fin on a un truc qui est hyper personnalisé et évolutif, mais qui au final peut-être n'a plus de sens » et selon M1 «il ne faut pas que le visiteur se perde, il faut qu'il reste focus sur ce qu'il veut voir. ». Pour plusieurs médiateurs, un système de recommandation d'œuvres sur la base des œuvres déjà appréciées ${ }^{13}$ se situe en deçà du rôle d'ouverture et de découverte que le musée doit tenir : « on va te contenir dans un truc que tu aimes, on ne va jamais te surprendre » (R2). Ils ont de plus exprimé leur méfiance envers de tels outils de surveillance et de recueil implicite des comportements des visiteurs.

Nous avons extrait de ces entretiens les principales caractéristiques selon lesquelles les visites guidées sont actuellement adaptées, ainsi que celles imaginées pour des visites personnalisées futures suivies en autonomie. Les principaux critères soulignés par plusieurs médiateurs sont : la thématique, l'âge, le niveau d'expertise, le temps disponible, la situation de handicap, la quantité de contenu, l'humeur (apprendre, jouer, collaborer, etc.) et la composition du groupe (famille, amis, seul, etc.). Le temps passé et la quantité de contenu peuvent être reliés aux quatre profils de visite (fourmi, papillon, sauterelle et poisson) de Levasseur et Veron (1983: 29-32), qui prennent en compte le nombre d'arrêts et le type de chemin suivi lors de visites libres. La motivation, l'expertise et la composition du groupe se retrouvent également dans les propriétés des cinq profils types de Falk (2009 : 157). Il est cependant nécessaire d'aller audelà de ces stéréotypes avec des profils utilisateurs plus riches et plus individuels pour atteindre une véritable personnalisation (Walsh et al., 2016).

\footnotetext{
${ }^{13}$ Comme dans les projets PEACH et CHIP décrits dans la première partie.
} 
Nous proposons ainsi de combiner entre elles les valeurs des critères évoqués ci-dessus afin d'affiner les profils des visiteurs au-delà de simples personas et de démultiplier ainsi les scénarios de visite possibles. Un groupe d'étudiants experts disposant d'une demi-journée et ayant envie d'apprendre en s'amusant ne visitera pas de la même manière qu'une famille avec un enfant de de sept ans en une heure souhaitant expérimenter. Une telle solution doit cependant prendre garde à conserver la cohérence de la visite, de ne pas enfermer les visiteurs dans ce qu'ils connaissent déjà et de respecter leur vie privée. Elle nécessite en outre la création d'un grand nombre de parcours de visite en amont.

\section{Besoins et exigences des médiateurs culturels pour la création de parcours personnalisés}

Les musées partenaires ne conçoivent actuellement pas de visites personnalisées au sens multicritères évoqué ci-dessus, or cela induit des défis différents de ceux du processus courant de création de visites guidées thématisées ou profilées. La combinaison des caractéristiques précédemment évoquées se traduit par des milliers de profils, et donc un nombre élevé potentiel de visites à créer. Un outil numérique est indispensable pour aider les médiateurs dans cette création. En vue de concevoir un dispositif adapté à leurs attentes, nous avons observé leurs stratégies et recueilli leurs besoins lors de deux exercices prospectifs de création de parcours personnalisés multicritères. Le premier a eu lieu au Musée Saint-Raymond (MSR) avec la directrice du service des publics ( $\mathrm{R}_{\mathrm{MSR}}$ ), et le second à la Cité du Vin (CDV) avec la responsable de la médiation culturelle $\left(\mathrm{R}_{\mathrm{CDV}}\right)$ et la responsable scientifique $\left(\mathrm{S}_{\mathrm{CDV}}\right)$, qui ont l'habitude de travailler ensemble sur l'exposition permanente. Nous avons préalablement validé avec les professionnelles des deux musées les caractéristiques des visiteurs collectées lors de l'étape précédente. Nous avons également défini leurs valeurs pour le contexte spécifique de chaque institution (les durées de visites possibles ne sont pas les mêmes pour un petit et un grand musée). Nous leur avons demandé de se placer dans des conditions écologiques (i.e. au plus près de leurs conditions de travail quotidiennes) afin de créer un maximum de visites personnalisées en deux heures environ. Après chaque observation, une discussion ouverte a été menée afin de recueillir des commentaires et retours qualitatifs. Ces exercices ont été filmés et transcrits sous forme de scénarios, de diagrammes de tâches et d'arbres d'exploration.

Au MSR, les valeurs des caractéristiques ont été choisies au cours de réunions préparatoires préalables à l'exercice. La responsable des publics a également éliminé les profils non pertinents (par exemple, les profils d'enfant de 3 à 6 ans seuls) et choisi un sous-ensemble de profils à traiter pour l'exercice (apprendre, sans handicap, débutant). À partir de ces informations, nous avons préparé une grille de profils avec toutes les combinaisons possibles pour suivre sa progression. L'espace de conception comprenait 1680 profils réalistes et 30 visites ont été créées en deux heures pour un thème choisi (« la mythologie »). La responsable des publics avait également réfléchi à l'avance son espace de création : elle avait amené des feuilles A3 et des post-it à accrocher sur les portes d'une armoire pour la création des parcours. Elle a noté l'importance pour elle d'être debout pour créer et de manipuler des objets physiques «j'ai besoin de faire le geste, de bouger les choses ». Elle a pris des photos des post-it positionnés au fur et à mesure et déclare qu'elle se souviendra de ce qu'elle a voulu faire et des liens entre les œuvres, car elle a manipulé les post-it « quand on voit tout devient plus clair, car matérialisé », "c'est mieux qu'une liste, la matérialité, le truc qu'on peut prendre et déplacer c'est important ». Les professionnelles de la CDV n'ayant pas eu le temps de participer à des réunions préparatoires, les caractéristiques ont été choisies juste avant l'exercice de création. Aucune grille représentant les combinaisons possibles n'a donc pu être préparée à l'avance. La présence de ce support est un biais qui doit être pris en compte dans la validité de nos résultats. De même, $\mathrm{R}_{\mathrm{MSR}}$ a eu plus de temps pour réfléchir à sa stratégie pour 
ce nouveau type de tâche que $R_{\mathrm{CDV}}$ et $\mathrm{S}_{\mathrm{CDV}}$. L'espace de conception de la CDV comprenait 11 136 profils possibles et 17 visites ont été créées en 1 heure et 40 minutes pour un thème (« les incontournables »).

Dans les deux cas, le processus de création a commencé par le choix d'une thématique et d'un ensemble d'œuvres correspondant. Elles ont ensuite choisi un à un les profils visiteurs pour lesquels elles ont créé une visite en sélectionnant un sous-ensemble d'œuvres ordonnées selon la logique de narration et leur emplacement dans le musée. Ce procédé était itératif, elles ont parfois copié ou dérivé une visite déjà créée pour un nouveau profil (une visite d'une heure peut être créée en enlevant certaines œuvres d'une visite d'une heure et demie pour le même type de visiteurs). Bien que cette approche générale soit commune aux expertes des deux musées, elles ont divergé dans leur façon d'explorer l'espace de conception. R $\mathrm{R}_{\mathrm{MSR}}$ a fixé toutes les contraintes (apprendre + sans handicap + débutant + adulte + une heure + deux personnes), constituant ainsi un profil complet, et a ensuite décidé d'une histoire à raconter pour celui-ci. Tandis que $\mathrm{R}_{\mathrm{CDV}}$ et $\mathrm{S}_{\mathrm{CDV}}$ ont fixé une seule contrainte (le nombre de personnes) et créé un parcours de base pour chacune des valeurs possibles (1 personne, 2 personnes, 3-6 personnes). Elles ont ensuite fixé une nouvelle contrainte (l'âge) et dérivé la base pour toutes les nouvelles valeurs (une personne de 3-6 ans, une personne de 7-12 ans, etc., deux personnes de 3-6 ans, deux personnes de 7-12 ans, etc.) en fonction de leur impact sur le parcours (contraintes spatiales, coopération potentielle, contenu non adapté). Elles ont refait cette étape pour toutes les contraintes, en reprenant le parcours créé précédemment comme base. Ainsi, les stratégies adoptées pour faire face à la complexité combinatoire sont différentes. L'une se concentre sur un profil complet pour lequel elle crée un contenu le plus original possible ( Si tu ne fais que décliner tu n'es plus dans la personnalisation. Ce qui est exaltant c'est de proposer des trucs particuliers », $\mathrm{R}_{\mathrm{MSR}}$ ), tandis que les autres se focalisent successivement sur une seule caractéristique pour laquelle elles déclinent par différenciation («ce qui est intéressant c'est la systématisation», $\mathrm{S}_{\mathrm{CDV}}$ ). Ces stratégies peuvent être influencées par les biais mentionnés ci-dessus (temps de réflexion, fourniture d'une grille de profils), mais leur diversité est tout de même intéressante à considérer.

Nous avons également observé que $\mathrm{R}_{\mathrm{MSR}}$ se recentrait régulièrement sur les valeurs des caractéristiques relatives aux visiteurs déjà choisies et celles qui lui restaient à faire. Elle consultait régulièrement la grille des profils, non seulement pour voir ses progrès, mais aussi comme rappel, aide à la décision, ou encore source d'inspiration. Par exemple, en voyant la valeur « expert » lors de la création d'une visite pour deux adultes débutants, elle a pensé à organiser un débat sur un tableau représentant l'enlèvement de Proserpine. À la CDV, où la grille n'était pas fournie, les responsables ont ressenti le besoin de créer un support pour voir l'ensemble des possibilités et suivre leur progression. Elles ont utilisé le tableur Excel pour créer le tableau de la Figure 3. Elles ont ajouté des sous-colonnes chaque fois qu'elles considéraient une nouvelle caractéristique. Par exemple, elles ont subdivisé chaque colonne existante pour «nombre de personnes » en autant de sous-colonnes que de valeurs possibles pour « âge » (3-6 ans, 7-12 ans, etc.). Mais la manipulation d'un tel tableau n'a pas été facile et elles ont exprimé le besoin d'un outil dédié. « Nous avons besoin de matrices avec 12000 entrées, nous avons besoin de quelque chose qui nous permette de voir en 3D » a déclaré la responsable scientifique. Dans les deux cas, elles ont ressenti le besoin d'un outil pour explorer leur espace de conception, composé de tous les profils visiteurs possibles.

Les expertes se sont déclarées très intéressées par cette démarche systématique permettant de se centrer et se projeter sur les profils et envies des visiteurs. $\mathrm{R}_{\mathrm{MSR}}$ a déclaré que les contraintes stimulaient sa créativité «je trouve que c'est bien parce que ça démultiplie la créativité, c'est une contrainte de plus qu'on s'inflige », tandis que $\mathrm{R}_{\mathrm{CDV}}$ et $\mathrm{S}_{\mathrm{CDV}}$ ont plutôt loué le côté systématique de la démarche «ce qui est intéressant c'est la systématisation », 
« c'est contraignant, mais ce que je trouve intéressant, c'est que ça oblige à se poser des questions que peut être on ne se poserait pas en allant trop vite sur le contenu, ou trop en détail sur le contenu, ou en allant trop sur la forme ». Une limitation majeure identifiée est la difficulté de créer un aussi grand nombre de parcours, d'où la nécessité de mettre en place un outil numérique permettant des stratégies de regroupement et de déclinaison, voir même de création automatique pour les profils moins prioritaires en s'inspirant de ce qui a été fait pour les autres profils.

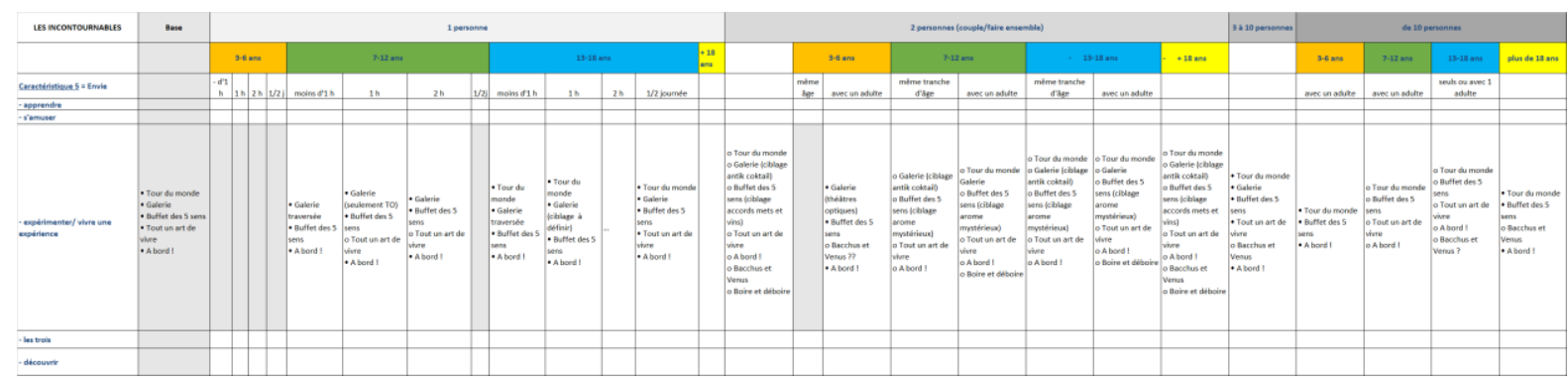

Figure 3 : Visites créées pendant l'exercice d'observation à la CDV, extrait du tableau créé par les utilisatrices

L'analyse de l'activité prospective de création de parcours de visite personnalisés multicritères dans deux institutions a fait émerger plusieurs exigences pour un tel outil : 1) le système doit permettre d'explorer l'espace de conception constitué de tous les profils de visiteurs, afin de voir ce qui a été fait et ce qui reste à faire ; 2) le système doit permettre aux médiateurs de se projeter sur le profil des visiteurs afin d'imaginer des parcours de visite adaptés ; 3) le système doit supporter plusieurs stratégies de création, les experts peuvent ainsi choisir de créer des visites originales pour des profils très spécifiques ou des visites de base pour des profils génériques qu'ils affinent ensuite de manière itérative.

Les observations nous ont également permis de dégager quelques recommandations. Premièrement, le travail de création de parcours de visite peut se faire à plusieurs et deuxièmement, certains professionnels ont besoin de manipuler physiquement les objets d'intérêt. La collaboration et la manipulation directe - voire tangible - sont donc des éléments à prendre en compte lors de la conception d'un outil d'aide à la création de parcours de visite personnalisés.

\section{Conclusion et perspectives}

Cette étude nous a permis de préciser ce que la notion de personnalisation pourrait recouvrir dans la sphère muséale, et ce que le numérique pourrait y apporter. Nous passons ainsi de scénarisations développées selon une thématique ou adaptées selon des profils génériques à une personnalisation multicritères prenant en compte les multiples facettes des visiteurs et leurs attentes renouvelées. Ces parcours de visite à la carte se positionnent ainsi dans une démarche d'accompagnement des visiteurs, afin de les aider à trouver ce qui dans les collections leur correspond, et ce en fonction de leurs différentes situations de visite. Les dispositifs évoluent donc pour passer d'une exigence de transmission culturelle posée par les professionnels des musées vers des orientations étroitement liées aux visiteurs et à leurs envies.

Les enjeux de telles visites sont nombreux, elles doivent par exemple maintenir une cohérence et un sens dans le contenu culturel présenté. Elles doivent également permettre d'ouvrir le regard du visiteur à de nouveaux savoirs et connaissances, sans le maintenir dans une «bulle de filtres" composée uniquement de ce qu'il aime déjà (Bakshi et al., 2015). La 
personnalisation pose également la question des données à caractère personnel, du consentement de leur partage et de leur exploitation, même à but non lucratif par un organisme culturel. Enfin, afin de s'adapter aux divers profils visiteurs, de nombreux parcours de visite doivent être créés en amont. C'est ici que le numérique peut apporter un premier appui, en permettant aux médiateurs de passer d'une création «artisanale» de parcours thématisés ou profilés, à une création « outillée » de parcours multicritères.

Dans cet article, nous faisons le pari d'augmenter les capacités des médiateurs plutôt que d'avoir recours à une intelligence artificielle permettant de générer les parcours. Pour autant les deux approches sont complémentaires et la génération automatique pourrait être une première étape pour des briques de parcours ou des parcours simples, à réutiliser et affiner par les médiateurs. Nos observations ont montré que pour créer de telles visites, les spécialistes ont besoin d'avoir une vision claire de leur espace de conception, composé de tous les profils visiteurs possibles, afin de mettre en place des stratégies de création de parcours pour ceux-ci. Cet espace de conception est vaste et multidimensionnel. Les différentes dimensions sont les caractéristiques du visiteur, telles que définies par l'analyse de l'activité ; auxquelles s'ajoute la dimension de complétude de la visite : parcours fait, à faire ou non pertinent pour un profil. La tâche d'exploration d'un tel espace est donc complexe et longue. Elle nécessite la création d'un outil permettant de visualiser le champ des possibles, de mettre en place une stratégie de choix des profils à traiter et de créer des parcours narratifs pour ceux-ci, voire de décliner automatiquement ces parcours pour les profils secondaires. Nos travaux de recherche portent sur la réalisation d'un tel outil, et nous avons ainsi commencé à explorer un espace de solutions pour la manipulation de la grille multicritères, tirant notamment parti des bénéfices des interactions tangibles, décrites ici pour les visiteurs, mais également applicable en contexte professionnel (Rey, Couture et al., 2020). Le deuxième point d'apport du numérique se situe dans la possibilité de restituer les parcours ainsi créés auprès des visiteurs, pour qu'ils choisissent celui qui leur correspond au mieux (Rey et al., 2018 ; Rey, Picard et al. 2020) mais également pour le suivre dans le musée sans que ce guidage prenne le pas sur leur expérience sensorielle du lieu et des expôts. Ce second aspect ouvre de nouvelles opportunités, telle que par exemple, la possibilité pour le visiteur d'adapter sa visite en temps réel en fonction de l'évolution de ses souhaits et envies. Selon Mariani-Rousset «le parcours est à la croisée des chemins entre le visiteur et le concepteur : c'est l'utilisation par l'un de l'espace organisé par l'autre» (2001 : 29-44). La personnalisation des parcours ouvre des possibilités en termes d'intersubjectivité, le parcours personnalisé devenant vecteur de transmission entre l'intention des concepteurs de l'exposition et l'expérience de visite des visiteurs.

\section{Bibliographie}

Adlin (Tamara) \& Pruitt (John). 2010. The Essential Persona Lifecycle: Your Guide to Building and Using Personas. Burlington : Morgan Kaufmann.

Andreacola (Florence). 2014. "Musée et numérique, enjeux et mutations ». Revue Française Des Sciences de l'information et de La Communication, 5, mis en ligne le 21 juillet 2014, consulté le 19 février 2020. URL : http://journals.openedition.org/rfsic/1056.

Antoniou (Angeliki) \& Lepouras (George). 2010. « Modeling Visitors' Profiles: A Study to Investigate Adaptation Aspects for Museum Learning Technologies ». Journal on Computing and Cultural Heritage (JOCCH), 3(2), p. 7:1-7:19. 
Ardissono (Liliana), Kuflik (Tsvi) \& Petrelli (Daniela). 2012. «Personalization in cultural heritage: the road travelled and the one ahead». User Modeling and User-Adapted Interaction, 22 (1-2), p. 73-99.

Arnaldi (Bruno), Guitton (Pascal) \& Moreau (Guillaume). 2018. Réalité virtuelle et réalité augmentée : Mythes et réalités. ISTE éditions.

Bakshy (Eytan), Messing (Solomon) \& Adamic (Lada A.). 2015. «Exposure to ideologically diverse news and opinion on Facebook ». Science, 348(6239), p. 1130 -1132.

Beaudouin-Lafon (Michel) \& Mackay (Wendy). 2003. «Prototyping tools and techniques », p. 106-1031 in The human-computer interaction handbook. New York: Associates, Lawrence Erlbaum.

Bougenies (Fanny), Houriez (Julie), Houriez (Simon) \& Leleu-Merviel (Sylvie). 2015. «Musée pour tous : un dispositif de découverte dans les murs et son évaluation », Culture \& musées, 26, p. 115-139.

Bowen (Jonathan P.) \& Filippini-Fantoni (Silvia). 2004. «Personalization and the Web from a museum perspective ». Museums and the Web.

Bradburne (James M.). 2001. «A new strategic approach to the museum and its relationship to society ». Museum Management and Curatorship, 19(1), p. 75-84.

Damala (Areti), Ruthven (Ian) \& Hornecker (Eva). 2019. «The MUSETECH Model: A Comprehensive Evaluation Framework for Museum Technology », Journal on Computing and Cultural Heritage, 12(1), p. 1-22.

Davallon (Jean). 2010. «L'écriture de l'exposition : expographie, muséographie, scénographie ». Culture \& Musées, 16(1), p. 229-238.

Eidelman (Jacqueline), Gottesdiener (Hana) \& Le Marec (Joëlle). 2013. «Visiter les musées : expérience, appropriation, participation ». Culture \& Musées, Muséologie et recherches sur la culture, (Hors-série), p. 73-113.

Falk (John H.). 2009. Identity and the Museum Visitor Experience. New York: Routledge.

Fenu (Cristina) \& Pittarello (Fabio). 2018. «Svevo tour: the design and the experimentation of an Augmented Reality application for engaging visitors of a literary museum ». International Journal of Human-Computer Studies, 114, p. 20-35.

Filippini-Fantoni (Silvia). 2009. Approche critique des stratégies de personnalisation dans les Musées : théorie et pratique. Thèse de Doctorat. Arts et sciences de l'art. Médiation de la culture. Paris 1 Panthéon-Sorbonne.

Gellereau (Michèle). 2005. Les mises en scène de la visite guidée : communication et médiation. Paris : L’Harmattan.

Mariani-Rousset (Sophie). 2001. «Espace public et publics d'expositions. Les parcours : une affaire à suivre », p. 29-44 in Espace urbain en méthodes / sous la direction de Michèle Grosjean \& Jean-Paul Thibaud. Marseille : Parenthèses, coll. Eupalinos.

Hewett (Thomas T.), Baecker (Ronald), Card (Stuart), Carey (Tom), Gasen (Jean), Mantei (Marilyn), Perlman (Gary), Strong (Gary) \& Verplank (William). 1992. ACM SIGCHI curricula for human-computer interaction. ACM.

Hood (Marilyn G). 1983. «Staying away: why people choose not to visit museums ». Museum news, 61(4), p. 50-57. 
Hornecker (Eva), Buur (Jacob). 2006. « Getting a grip on tangible interaction ». Proceedings of the SIGCHI Conference on Human Factors in Computing Systems, p. 437 - 446.

Hornecker (Eva) \& Ciolfi (Luigina). 2019. Human-Computer Interactions in Museums. Synthesis Lectures on Human-Centered Informatics. Morgan \& Claypool Publishers.

Hornecker (Eva), Stifter (Matthias). 2006. «Learning from Interactive Museum Installations About Interaction Design for Public Settings », Proceedings of the 18th Australia conference on Computer-Human Interaction: Design: Activities, Artefacts and Environments, p. 135-142.

Lallemand (Carine) \& Gronier (Guillaume). 2018. Méthodes de design UX: 30 méthodes fondamentales pour concevoir des expériences optimales. Paris : Eyrolles.

Levasseur (Martine) \& Veron (Eliseo). 1983. «Ethnographie d'une Exposition ». Histoires d'expo, Peuple et Culture, p. 29-32.

McHugh (Kelly). 2017. Museum and the Web 2017. GLAMi nomination. Consulté sur https://mw17.mwconf.org/glami/journeymaker/ le 22/01/2020.

Naudet (Yannick), Lykourentzou (Ioanna), Tobias (Eric), Antoniou (Angeliki), Rompa (Jenny) \& Lepouras (George). 2013. « Gaming and Cognitive Profiles for Recommendations in Museums ». Proceedings of the 8th International Workshop on Semantic and Social Media Adaptation and Personalization, p. 67-72.

Norman (Don A.) \& Draper (Stephen W.). 1985. User Centered System Design: New perspectives on Human-Computer Interaction. Hillsdale, New Jersey: Lawrence Erlbaum Associates.

Not (Elena) \& Petrelli (Daniela). 2018. «Blending customisation, context-awareness and adaptivity for personalised tangible interaction in cultural heritage ». International Journal of Human-Computer Studies, 114, p. 3-19.

Petrelli (Daniela), Ciolfi (Luigina), van Dijk (Dick), Hornecker (Eva), Not (Elena) \& Schmidt (Albrecht). 2013. "Integrating material and digital: a new way for cultural heritage». Interactions, 20(4), p. 58-63.

Petrelli (Daniela) \& O’Brien (Sienead). 2018. «Phone vs. Tangible in Museums: A Comparative Study », Proceedings of the SIGCHI Conference on Human Factors in Computing Systems, p. 1-12.

Rey (Stéphanie), Couture (Nadine), Picard (Célia), Bortolaso (Christophe), Derras (Mustapha) \& Brock (Anke M.). 2020. «Designing Tangible Tools for the Creation of Personalized Visits by Museum Professionals ». Proceedings of the fourteenth International Conference on Tangible, Embedded, and Embodied Interaction.

Rey (Stéphanie), Mauriéras (Pierre), Picard (Célia) \& Brock (Anke M.). 2018. « Totem de Personnalisation : Conception d'une Interface Tangible pour Choix de Parcours de Visite dans les Musées ». Proceedings de la 30ème conférence francophone sur l'Interaction HommeMachine, p. 219-226.

Rey (Stéphanie), Picard (Célia), Fatmi (Yanis), Franco (Fanny), Guilbert (Sarah), Manéré (Jérémy), Bortolaso (Christophe), Derras (Mustapha), Couture (Nadine) \& Brock (Anke M.). 2020. «Build Your Own Hercules: helping visitors personalize their museum experience. » Proceedings of the fourteenth International Conference on Tangible, Embedded, and Embodied Interaction. 
Roussou (Maria) \& Katifori (Akrivi). 2018. «Flow, Staging, Wayfinding, Personalization: Evaluating User Experience with Mobile Museum Narratives ». Multimodal Technologies and Interaction. Multidisciplinary Digital Publishing Institute, 2(2), p. 32.

Roussou (Maria), Katifori (Akrivi), Pujol (Laia), Vayanou (Maria) \& Rennick-Egglestone (Stefan J). 2013. «A life of their own: museum visitor personas penetrating the design lifecycle of a mobile experience ». Extended Abstracts on Human Factors in Computing Systems, p. 547-552.

Shaer (Orit) \& Hornecker (Eva). 2010. Tangible User Interfaces: Past, Present, and Future Directions. Hanover, Hanover, MA, USA: Now Publishers Inc.

Stock (Oliviero), Zancanaro (Massimo), Busetta (Paolo), Callaway (Charles), Krüger (Antonio), Kruppa (Michael), Kuflik (Tsvi), Not (Elena) \& Rocchi (Cesare). 2007. « Adaptive, intelligent presentation of information for the museum visitor in PEACH ». User Modeling and User-Adapted Interaction 17(3), p. 257-304.

Vayanou (Maria), Katifori (Akrivi), Karvounis (Manos), Kourtis (Vassilis), Kyriakidi (Marialena), Roussou (Maria), Tsangaris (Manolis), Ioannidis (Yannis), Balet (Olivier), Prados (Thibaut), Keil (Jens), Engelke (Timo) \& Pujol (Laia). 2014. " Authoring personalized interactive museum stories ». Proceedings of the International Conference on Interactive Digital Storytelling, p. 37-48. Springer, Cham.

Walsh (David), Clough (Paul) \& Foster, (Jonathan). 2016. «User categories for digital cultural heritage ». First International Workshop on Accessing Cultural Heritage at Scale.

Wang (Yiwen), Stash (Natalia), Aroyo (Lora), Gorgels (Peter), Rutledge (Lloyd) \& Schreiber (Guus). 2008. «Recommendations based on semantically enriched museum collections ». Web Semantics: Science, Services and Agents on the World Wide Web, 6(4), p. 283-290.

Wecker (Alan J.), Kuflik (Tsvi) \& Stock (Oliviero). 2016. «Dynamic personalization based on mobile behavior ». Proceedings of the 18th International Conference on Human-Computer Interaction with Mobile Devices and Services Adjunct, p. 978-983. 